1

\title{
Cell-matrix adhesion contributes to permeability control in human colon organoids
}

(1)

(1)

6

By

James Varani, Shannon D. McClintock and Muhammad N. Aslam*

${ }^{*}$ Corresponding author:

Muhammad N. Aslam

Department of Pathology

The University of Michigan

1150 W Medical Center Dr

Ann Arbor, MI 48109, USA

Tel: (734) 936-1897

Email: mnaslam@med.umich.edu

The Department of Pathology

The University of Michigan Medical School

Ann Arbor, Ml 48109

From 


\section{ABSTRACT}

31 Background and aims. Cell-cell adhesion structures (desmosomes and, especially, tight

32 junctions) are known to play important roles in control of transepithelial permeability in the colon.

33 The involvement of cell-matrix interactions in permeability control is less clear. The goals of the

34 present study were to: i) determine if disruption of colon epithelial cell interactions with the extracellular matrix alters permeability control and ii) determine if increasing the elaboration of protein components of cell-matrix adhesion complexes improves permeability control and

37 mitigates the effects of cell-matrix disruption.

Methods. Human colon organoids were interrogated for transepithelial electrical resistance (TEER) under control conditions $(0.25 \mathrm{mM}$ calcium $)$ and in the presence of Aquamin ${ }^{\circledR}$, a multimineral product, at a level providing $1.5 \mathrm{mM}$ calcium. The effects of Aquamin ${ }^{\circledR}$ on cell-matrix adhesion protein expression were determined in a proteomic screen and by Western blotting. In parallel, TEER was assessed in the presence of a function-blocking antibody directed at an epitope in the C-terminal region of laminin $\alpha 3$ chain.

Results. Treatment of colon organoids with Aquamin ${ }^{\circledR}$ increased the expression of multiple basement membrane and hemidesmosomal proteins as well as keratin 8 and 18. TEER values were higher in the presence of Aquamin ${ }^{\circledR}$ than they were under control conditions. Anti-laminin

47 antibody reduced TEER values under all conditions but was most effective in the absence of

48 Aquamin $^{\circledR}$, where laminin expression was low and TEER values were lower to begin with.

Conclusions. These findings provide evidence that cell-matrix interactions contribute to permeability control in the colon. They suggest that the elaboration of proteins important to cell-

51 matrix interactions can be increased in human colon organoids by exposure to a multi-mineral

52 natural product. Increasing the elaboration of such proteins may help to mitigate the

53 consequences of disrupting cell-matrix interactions on permeability control. 


\section{INTRODUCTION}

Functional defects in the gastrointestinal tract barrier have been documented in inflammatory conditions of the bowel, including both ulcerative colitis (UC) and Crohn's Disease

57 (1-5). Barrier defects have also been described in irritable bowel syndrome (6) and noted in celiac disease (5) and as a consequence of acute bacterial infection (7). Barrier defects have also been seen in obesity related to high-fat and high-sugar diets $(8,9)$ and, thus, may contribute to chronic, systemic inflammation. Finally, gastrointestinal discomfort associated with chronic environmental stress may reflect barrier dysfunction related to corticosteroid release (10). In these situations, inflammatory injury to the intestinal wall contributes to barrier breakdown. At the same time, however, pre-existing barrier defects, leading to permeation of bacteria, bacterial products, food allergens and toxins into the mucosal wall, may promote inflammation in the gastrointestinal tract (4).

Tight junctions are thought to be the epithelial cell surface structures that most directly contribute to permeability control - at least in so far as soluble factors are concerned (11-17).

Desmosomes are also important, if for no other reason than that they are largely responsible for tissue cohesion and strength (11-15,18-20). It is difficult to envision effective control of permeability in a mechanically-active tissue such as the colon if tissue cohesion is compromised. In support of this, we recently demonstrated that Aquamin ${ }^{\circledR}$, a calcium- and magnesium-rich, multi-mineral product obtained from marine red algae (21), strongly upregulated desmosomal proteins and increased the number of desmosomes in human colon tissue in organoid culture but had little effect on tight junctional elements (22-24). In parallel with these desmosomal changes, both permeability control and tissue cohesion were increased (23). The same multi-mineral intervention that increased desmosomes also up-regulated expression of other moieties that contribute to the permeability barrier. Among these were 
cadherin family members, carcinoembryonic antigen cell adhesion molecules (CEACAM),

79 mucins and trefoils.

In the present study, we have used a proteomic screen to assess the expression of

81 proteins involved in cell-matrix interactions in human colon organoid culture. Studies by other

82 investigators have utilized immunohistochemical methods to show basement membrane defects

83 in UC and Crohn's disease as well as in other inflammatory conditions of the bowel (25-28).

84 While these findings suggest a role for cell-basement interactions in barrier function, how these

85 interactions influence gastrointestinal permeability, per se, has not been studied. Here it is

86 demonstrated that proteins affecting cell-basement membrane interactions through both focal

87 adhesions and desmosomes are increased in response to Aquamin ${ }^{\circledR}$. Further, it is shown that

88 an antibody to the major cell adhesion domain in the laminin $\alpha$-chain decreases transepithelial

89 electrical resistance (TEER) - i.e, a surrogate for permeability control - in human colon

90 organoid culture. In contrast, treatment with the same antibody has no apparent effect on tissue

91 cohesion / tissue strength. 


\section{MATERIALS AND METHODS}

Aquamin $^{\circledR}$. This is a calcium-rich, magnesium-rich, trace element-rich multi-mineral product obtained from the skeletal remains of the red marine algae, Lithothamnion sp (21) (Marigot Ltd, along with measurable levels of 72 other trace minerals (essentially all of the trace elements algae fronds accumulate from the deep ocean water). The same single batch of Aquamin ${ }^{\circledR}$ Soluble that was used in the previous colon organoid studies (22-24) was used for this study. File S1 describes the complete mineral/trace element composition of the multi-mineral product - Aquamin ${ }^{\circledR}$. region in the C-terminal end of the molecule made up of 5 modules (referred to as Laminin $\mathrm{G}$ domain-like modules). Cell-binding sites are present within these modules (29-31). A mouse monoclonal antibody (IgG1 clone) prepared against an epitope within the G domain of the human laminin $\alpha 3$ chain was used for functional blockade. This antibody (clone \#P3H9-2; R\&D Systems) has been demonstrated to detect antigen in a variety of epithelia and has been shown to inhibit cell proliferation of both rat and human epithelial cells (32). A control mouse monoclonal lgG1 immunoglobulin was used in parallel with the anti-laminin antibody for comparison. A rabbit polyclonal antibody (Invitrogen; PA5-27271) prepared against a was used as control.

112 Organoid culture: Colon tissue in organoid culture was available from our previous studies (22-

113 24). The Institutional Review Board at the University of Michigan Medical School (IRBMED)

114 approved the tissue collection and use protocol. Subjects provided written informed consent 115 prior to flexible sigmoidoscopy and biopsy collection. This study was conducted according to the 
principles stated in the Declaration of Helsinki. For the present work, cryopreserved colon organoid samples were put into culture and expanded over a 3-4 week period. After expansion, organoids were used to assess transepithelial electrical resistance (TEER) (below) or tissue cohesion (below). Additional organoid samples were used for Western blotting. Three media formulations were used in this study. These include: and the same base media that had been conditioned by the previous growth of L-cells engineered to provide a source of recombinant Wnt3a, R-spondin-3, and Noggin - referred to as L-WRN conditioned medium (33). The growth medium was supplemented with $100 \mathrm{ng} / \mathrm{mL}$ human recombinant epidermal growth factor (EGF) (R\&D) as the major growth-supporting peptide and was further supplemented with $10 \mu \mathrm{M}$ Y27632 (Tocris), 500 nM A83-01 (Tocris),

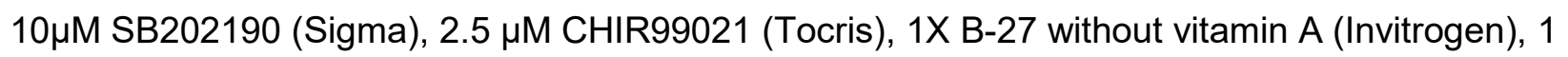
mM N-Acetyl-L-cysteine, 10 mM HEPES (Invitrogen), 2 mM Glutamax (Invitrogen), $10 \mu \mathrm{M}$ SB202190 (Sigma), and $100 \mu \mathrm{g} / \mathrm{mL}$ Primocin (InvivoGen). Since L-WRN medium was supplemented with $20 \%$ fetal bovine serum, after $1: 1$ dilution, the final concentration of the growth medium was $10 \%$ serum. and F12 media. This formulation lacked Wnt3a and R-spondin-3 but was supplemented with EGF (50 ng/mL) along with Gastrin (10 nM, Sigma), Noggin (50 ng/mL, R\&D), and Y27632 (2.5 
dilution), the serum concentration was $2.5 \%$ and the calcium concentration was $0.25 \mathrm{mM}$ (and used as a control). When Aquamin ${ }^{\circledR}$ was included, it was added at $0.51 \mathrm{mg} / \mathrm{mL}$; an amount to

142 bring the final calcium concentration to $1.5 \mathrm{mM}$.

Assessment of TEER. Organoids were dissociated into small cell aggregates (less than $40 \mu \mathrm{m}$ in size) and plated onto collagen IV (Sigma)-coated transwells $\left(0.4 \mu \mathrm{m}\right.$ pore size, $0.33 \mathrm{~cm}^{2}, \mathrm{PET}$, Costar) at 200,000 individual organoids per well in growth medium as in Zou et al. (34). After seeding in growth medium, organoids were allowed to attach to the TEER membrane and incubated without further treatment for one day. Then growth medium was replaced with either differentiation medium or with the growth medium-KGM Gold mix with or without Aquamin ${ }^{\circledR}$.

The function-blocking anti-laminin antibody was included at the start of the treatment period at $25 \mu \mathrm{g} / \mathrm{mL}$ in each of the culture media. Fresh culture medium and antibody was provided every two days during the assay period. A control mouse IgG was used at the same concentration for comparison. TEER values were determined daily beginning on day-2 using an epithelial volt/ohm meter (EVOM2, World Precision Instruments) and STX2 series chopstick electrodes as described previously (23).

Histochemical staining and light microscopy. At the completion of TEER measurements on day2 and day-5), membranes with cells still attached were prepared for light microscopy. The membranes were fixed for 1 hour in $10 \%$ buffered formalin. Following this, membranes were paraffin-embedded, sectioned and stained with hematoxylin and eosin. The stained specimens were visualized by light microscopy. Slides were digitally scanned using the Aperio AT2 brightfield whole slide scanner (Leica Biosystems) at a resolution of $0.5 \mu \mathrm{m}$ per pixel with $20 \mathrm{X}$ objective.

Western blotting. At the completion of the TEER measurements, organoids were harvested for protein. Briefly, wells were washed gently with PBS, then harvested using $0.4 \%$ EDTA to 
dissolve and remove matrigel. The remaining pellet containing organoids was then washed 3 times with PBS and subjected to extraction using RIPA buffer (89901; Thermo Scientific). Organoids were lysed by repetitive pipetting in the buffer, followed by incubation for 10 minutes on ice. Non-soluble cellular debris was removed by centrifugation at $14,000 \mathrm{~g}$ for 10 minutes and protein was quantified using a BCA assay (23227; Pierce). Samples were heated for 10 minutes at $70^{\circ} \mathrm{C}$ in NuPage LDS sample buffer and then run on 3-8\% Tris-Acetate gels using NuPage MOPS running buffer under reducing conditions. Proteins were then transferred onto

171 nitrocellulose membranes, blocked with $5 \%$ non-fat dry milk and probed with the primary and 172 appropriate secondary antibodies. Secondary antibodies were used at 1:5000 for all

173 membranes. $\beta$-actin was used as a loading control in each assay. SuperSignal WestPico Plus

174 (34577; Thermo Scientific) detection reagent was used and bands were visualized using a 175 BioRad ChemiDoc XRS+ Molecular Imager. Relative band density was determined using 176 Image $\mathrm{J}$ gel analysis tools.

177 Confocal fluorescence microscopy. At the completion of the TEER assay, membranes were prepared for confocal fluorescence microscopy and stained with an antibody to occludin for the 179 purpose of visualizing the cell layer. The membranes with cells still attached were fixed for 15 minutes at $-20^{\circ} \mathrm{C}$ in methanol. They were then washed three times in PBS before blocking in 3\% BSA (A8806; Sigma) in PBS for 1 hour. Following this, membranes were stained with an 182 antibody to occludin (331594; Invitrogen) 1:400 for 1 hour in 1\% BSA in PBS. Stained membranes were rinsed 3 times ( 5 minutes each) in PBS, stained with DAPI for 5 minutes to 184 identify nuclei and washed an additional 3 times with PBS. Finally, the membranes were gently 185 cut from the transwell insert and mounted apical side up on Superfrost Plus glass slides (Fisher 186 Scientific, Pittsburgh, PA) with Prolong Gold (P36930; Life Technologies Molecular Probes).

187 The stained specimens were visualized and imaged with a Leica Inverted SP5X Confocal 188 Microscope System (University of Michigan Medical School Biomedical Research Core Facility). 
Organoid cohesion assay. Organoid cohesion was assessed as described previously (23).

Briefly, after establishment and culture expansion, organoids were incubated in growth medium-

KGM Gold with or without the same anti-laminin antibody $(25 \mu \mathrm{g} / \mathrm{mL})$ as described above.

192 Treatment was for seven days with fresh medium and antibody added at days 2 and 4 . At the end of the incubation period, phase-contrast microscopy (Hoffman Modulation Contrast Olympus IX70 with a DP71 digital camera) was used to capture images in order to measure the size of multiple individual organoids (53-104 individual organoids per condition). Then organoids were then separated from the Matrigel and fragmented with mechanical force alone by pipetting the entire pellet 30x through an uncut 200 microliter pipet tip. After washing $3 x$ in PBS, organoids were re-cultured in fresh Matrigel. One day after establishment, multiple colonoids (colon organoids) were again examined under phase-contrast microscopy and sized. For both pre-harvest and post-harvest samples, phase-contrast images were analyzed using area measurements in Adobe Photoshop (CC version 19.1.5). Average organoid size-reduction

202 (fold-change before and after harvest and mechanical fragmentation) was determined by dividing the average post-harvest surface area by the average pre-harvest area.

204 Differential proteomic analysis: Proteomic assessment was conducted at the Proteomics 205 Resource Facility (PRF) in the Department of Pathology at the University of Michigan using

207 complete details for the experimental conditions, protocols and analysis methodology can be

208 found in previously published reports $(22,24)$. Briefly, fifty micrograms of organoid protein from

209 each condition were digested with trypsin and individually labeled with isobaric mass tags.

210 Labeled peptides were fractionated using 2D-LC (basic $\mathrm{pH}$ reverse phase separation followed

211 by acidic $\mathrm{pH}$ reverse-phase) and analyzed on a high-resolution, tribrid mass spectrometer

212 (Orbitrap Fusion Tribrid, ThermoFisher Scientific) using conditions optimized in the PRF.

213 MultiNotch MS3 was employed to obtain accurate quantitation of the identified 
214 proteins/peptides. Data analysis involved peptide filtering to retain only those that passed $\leq 2 \%$

215 false discovery rate (FDR) threshold of detection. Quantitation was performed using high-quality

216 MS3 spectra. Differential protein expression values (fold-change) for proteins of interest in each

217 treatment group were compared to protein values of the respective control group. Proteins were

218 identified using Universal Protein Resource (Uniprot) databases (Uniprot.org). Reactome

219 version 75 - a pathway analysis database was used to recognize associated pathways for

220 species "Homo sapiens" (reactome.org). For the purpose of the present study, we accessed

221 two existing data sets - one consisting of colon organoid tissue from four healthy subjects and

222 the other consisting of colon organoid tissue from three ulcerative colitis patients in remission.

223 In all cases, organoids grown in the growth medium-KGM Gold mix were compared to

224 organoids grown in the same medium supplemented with Aquamin ${ }^{\circledR}$ at levels providing 1.5 to

$2253.0 \mathrm{mM}$ calcium. For comparison purposes, the data presented here include only the maximum

226 response. The complete proteomics data sets are available at the ProteomeXchange

227 Consortium via the PRIDE partner repository with the dataset identifier PXD020244 for UC

228 derived colon organoids (and identifier pending for normal colon organoids).

229 Statistical analysis. Means and standard deviations were obtained for discrete values in each

230 assay (TEER, cohesion, and individual proteins from proteomics). Data generated in this way

231 were analyzed by ANOVA followed by paired t-test (two-tailed) for comparison using GraphPad

232 Prism version 8.3. 


\section{RESULTS}

234

235

236

237

238

239

240

241

242

243

244

245

Anti-laminin antibody reduces TEER values in organoids maintained in differentiation medium.

In the first series of studies, human colon organoids were plated on transwell membranes in growth medium. One day later, differentiation medium replaced growth medium and electrical resistance was assessed over subsequent days. Results are shown in Fig 1. Fig 1A demonstrates that under conditions optimized to promote electrical resistance (i.e., in differentiation medium), TEER values were low during the first two days after treatment, rose precipitously between days-3 and day-5, and fell thereafter. A combination of antibody to occludin (tight junctional protein) and DAPI (nuclear stain) was used to illuminate organoids on the membranes at day-2 and day-5. As shown in the inserts in Fig 1A, an intact cell-cell border could be seen in organoids by day-2. However, cell outgrowth from the organoids did not completely cover the membrane surface at this time (accounting for the lack of electrical resistance). Coverage of the membrane surface was complete by day- 5 .

Fig 1B demonstrates the effects of a function-blocking antibody to the $\alpha 3$ chain of laminin on electrical resistance in differentiation medium. A modest decrease in TEER values was observed at days-3, -4 and -5 (9-17\% decrease). When values from the three time-points were averaged together, the decrease observed with the anti-laminin antibody compared to the IgG control reached a statistically significant level (mean decrease $=11 \pm 3 \% ; p<0.01$ by 2 -tailed t-test).

At the completion of TEER assessment, membranes with cells still attached were fixed in $10 \%$ buffered formalin, stained with hematoxylin and eosin and examined at the light microscopic level (Fig 1B insert). It can be seen that under control conditions (lgG-treated cells) or in the same medium with anti-laminin antibody, most of the membrane surface was covered with a complete monolayer of cells. However, in the presence of the anti-laminin antibody, focal 
areas where cells had detached from the underlying substrate could be observed. In these areas, cell-cell attachments remained intact such the structure had the appearance of a tiny "blister."

261 hemidesmosome formation and keratins. Fig 2A presents data from the proteomic assessment 262 based on merged data from healthy subjects $(n=4)$ and subjects with $U C(n=3)$. In both data 263 sets (assessed independently), strong up-regulation of several laminin chains $(\alpha 1, \beta 1, \beta 2$ and $264 \gamma 1$ ) (components of laminin 111 and 121) along with nidogen-1 and the basement membranespecific heparin sulfate proteoglycan (HSPG-2, perlecan) was seen. Also detected in the proteomic analysis (Fig 2A) were laminin $\alpha 3, \beta 3$ and $\gamma 2$ chains (components of laminin 332 or

267 laminin-5 in the older terminology). This laminin isoform is a major component of hemidesmosomes $(35,36)$ and is considered as a skin-specific laminin isoform $(37)$. While these 269 laminin chains did not demonstrate an increase in response to Aquamin ${ }^{\circledR}$ in colon organoids, 270 several other hemidesmosomal proteins were detected, and a subset of these (plectin, 271 desmoplakin and epiplakin) were increased by Aquamin ${ }^{\circledR}$ as compared to control. The plakins 272 are critical linkers between laminin in the hemidesmosomes and intermediate filaments $(38,39)$.

274 keratin 8 and keratin 18 (components of intermediate filaments in gastrointestinal epithelial 275 cells) (40) were increased in response to Aquamin ${ }^{\circledR}$. With keratin 8, expression was increased $276 \quad 1.25 \pm 0.01$-fold and $1.52 \pm 0.09$-fold in the normal and UC data sets respectively. With keratin

27718 , values were $1.52 \pm 0.41$-fold and $1.86 \pm 0.00$-fold. Of interest, recent studies have

278 demonstrated that mutations in Keratin 8/18 in colonic epithelial cells are associated with loss of 279 permeability control in inflammatory bowel disease (41). Similarly, acute inflammation has been 280 shown to reduce Keratin 8/18 expression; levels were restored with improvement in disease 281 status as assessed by both clinical and endoscopic parameters (42). 
In addition to the findings presented above, certain other proteins of interest were also detected in the protein screen. The $\alpha 2$ chain of type IV collagen was detected; it was modestly

284 increased in response to Aquamin ${ }^{\circledR}$. A number of integrin components were also detected, but components of the major laminin-binding integrins $(\alpha 6, \beta 1$ and $\beta 4)$ were not significantly altered with Aquamin ${ }^{\circledR}$ as compared to control. Among other moieties that have been reported to

287 interact with laminin, both dystroglycan and syndecan were slightly down-regulated, sulfatide was unchanged and oncofetal antigen/immature laminin receptor OFA(iLRP)/67-kD laminin receptor was not detected. Finally, three proteins that serve as connectors between focal adhesions and the actin cytoskeleton (talin, vinculin and $\alpha$-actinin) were detected but did not 291 alter except vinculin, which was up-regulated in both sets (1.42- and 1.24-fold; in normal and 292 UC respectively). Finally, we searched for the pathways associated with the proteins 293 (presented in Fig 2A) using Reactome. Top 15 pathways with the involved entities are presented in Table 1. Type I hemidesmosome assembly, laminin interactions and extracellular matrix organization were at the top of the pathways list (Table 1).

Western blotting with an antibody to the laminin $\beta 1$ chain (most highly up-regulated of all the laminin chains detected in the proteomic screen) was used to confirm laminin up-regulation. Fig 2B shows the dramatic increase in laminin $\beta 1$ expression in Aquamin ${ }^{\circledR}$-treated organoids as compared to control.

$\underline{\text { TEER values in organoids: Effects of Aquamin }}{ }^{\circledR}$ and anti-laminin treatment. Human colon organoids were plated on transwell membranes in growth medium. One day later, growth medium was replaced with the growth medium-KGM Gold mix ( $0.25 \mathrm{mM}$ calcium; final concentration). In some wells, Aquamin ${ }^{\circledR}$ was added to bring the final calcium level to $1.5 \mathrm{mM}$ and provide the additional trace elements that make up the marine algae product. Electrical resistance across the cell layer was assessed as above. In the unsupplemented growth 
medium-KGM Gold mix, a TEER value of 1800 ohms per $\mathrm{cm}^{2}$ was achieved as compared to 2300 ohms per $\mathrm{cm}^{2}$ in differentiation medium (22\% decrease) while the TEER value in Aquamin ${ }^{\circledR}$ supplemented medium was virtually identical to that seen in differentiation medium

309 (compare values in Fig 3 with those in Fig 1A).

Fig 3 also shows the effects of anti-laminin treatment on TEER values in the two 311 conditions. In Aquamin-supplemented medium, TEER values were reduced by $16 \%$ with anti312 laminin. This is comparable to what was seen in differentiation medium (Fig 1B). In un313 supplemented growth medium-KGM Gold, where TEER values were lower to begin, the 314 inclusion of anti-laminin antibody further reduced TEER values by $57 \%$.

315 Effects of anti-laminin antibody on organoid cohesion. In our previous study, we demonstrated 316 that treatment of human colon organoids with Aquamin ${ }^{\circledR}$ increased organoid cohesion in parallel 317 with TEER (23). To determine whether interactions involving laminin contributed to intra318 organoid cohesion, organoids were maintained for a two-week period in growth medium-KGM 319 Gold with and without anti-laminin. At the end of the incubation period, cohesion was assessed 320 as described in Methods and in our previous study (23). As shown in Fig 4, no detectable 321 antibody effect on organoid cohesion was seen. It was evident that there was no difference in 322 the ratio of change in organoid size from post- to pre-harvest, the ratio was 0.45 and 0.46 with $323 \lg$ and anti-laminin antibody, respectively (Fig 4). 


\section{DISCUSSION}

Most studies of barrier dysfunction in the gastrointestinal tract have focused on the structural components that regulate cell-cell interactions (i.e., desmosomes and, especially, tight

327 junctions) (16-19), but basement membrane disruptions are also commonly observed (25-28).

328 Experimental animal models of colitis, likewise, demonstrate basement membrane disruptions in 329 inflamed colonic tissue $(28,43)$. In all of these settings, a loss or reduction in laminin 330 immunoreactivity is commonly observed (25-28), although altered distribution of laminin forms 331 has been reported as well, with some forms actually increasing (27). Laminin is not unique in 332 being altered in inflammatory bowel conditions. Basement membrane collagens including both 333 type IV and type $\mathrm{V}$ have been reported to be increased in inflamed bowel (28). Together, these 334 past findings provide a picture of widespread cell-matrix disruption in the context of the inflamed 335 colon. Although these changes are thought to be a consequence of the inflammatory process, 336 anomalies have been noted in some patients with inflammatory bowel disease in the absence of 337 acute tissue damage (4). Thus, pre-existing basement membrane irregularities may contribute 338 to inflammation, and not simply be the consequence of tissue injury. In support of this, murine 339 models in which laminin $\alpha$-chain was over-expressed showed a decreased sensitivity to 340 chemical-induced colitis (28). In another model, hemidesmosome disruption promoted colitis 341 (43) in genetically manipulated animals.

Regardless of whether preexisting barrier defects in the gastrointestinal tract promote 343 bowel inflammation or are simply the consequence of acute inflammation, improvement in 344 barrier structure / function would seem to be of value. The findings presented here allow us to 345 conclude i) that interfering with cell-basement membrane interactions reduces permeability 346 control without a major effect on tissue cohesion in human colon organoid culture; ii) that 347 treating colon organoids with a multi-mineral supplement leads to increased elaboration of 348 basement membrane proteins and hemidesmosomal / intermediate filament components; and 
iii) that the increased elaboration of these proteins mitigates, at least partially, the consequences of interfering with cell-basement membrane interactions. As summarized graphically in the cartoon (Fig 5), the basement membrane, hemidesmosomal and intermediate

352 filament proteins that are responsive to Aquamin ${ }^{\circledR}$ treatment could be expected to have an effect 353 on both focal adhesions and hemidesmosomes (44). In our previous studies, the same mineral 354 supplement was shown to substantially increase desmosomes along the lateral surface of 355 adjacent epithelial cells in colonoid culture without a major effect on tight junctions (22-24).

356 Together with the data presented in our earlier study, the findings shown here suggest that 357 while tight junctions maybe critical to permeability control in the gastrointestinal tract, both cellcell and cell-matrix interactions are also important.

The studies carried out here made use of a sophisticated ex vivo culture system, and whether the effects obtained in vitro have relevance to what occurs in vivo remains to be demonstrated. In an effort to begin addressing this issue, we have recently carried out a pilot phase trial in which ten healthy subjects were treated with the same multi-mineral product

$363\left(\right.$ Aquamin $\left.^{\circledR}\right)$ used here. To summarize the results of this pilot study, there were no tolerability 364 issues with daily Aquamin ${ }^{\circledR}$ ingestion over a 90-day period and no safety concerns (45). Equally 365 important, when Aquamin ${ }^{\circledR}$-treated subjects were compared to subjects receiving placebo for 366 the same period, we saw up-regulation of laminin chains along with increased levels of other 367 basement membrane components and hemidesmosome moieties in colonic biopsies (46). 368 Subjects receiving calcium alone (i.e., the most abundant mineral in Aquamin ${ }^{\circledR}$ ) also demonstrated increases in several of the same molecules, but the degree of up-regulation with calcium alone was lower than that seen with Aquamin ${ }^{\circledR}(46)$. 
374 proteomics) are being used to evaluate proteins changes in the colon over the course of

375 intervention. In parallel, the urine lactulose/mannitol ratio (47) is being assessed to provide a

376 direct measure of treatment effects on gastrointestinal permeability (ClinicalTrials.gov:

377 NCT04855799). If successful, Aquamin ${ }^{\circledR}$ or a similarly formulated product could be used as a

378 low-cost, low- to no-toxicity adjuvant therapy to improve gastrointestinal barrier function in

379 individuals suffering from a variety of gastrointestinal maladies. At the very least, individuals

380 with barrier defect-associated gastrointestinal conditions should be encouraged to include an

381 adequate source of calcium and other minerals in their diet. Unfortunately, deficiencies in

382 calcium and other critical mineral components are widespread throughout the world (48) and

383 this is especially true for those consuming a Western-style diet (49).

Finally, there is another group of diseases - epidermolysis bullosa and related

conditions - that are manifestations of mutations in various basement membrane, desmosomal / hemidesmosomal and keratin genes (50). At the same time, there are case reports and studies that provide evidence of an association between bullous pemphigoid and inflammatory bowel disease (51-53). At this point, we can only speculate as to whether optimizing the expression of multiple cell-cell and cell-matrix adhesion molecules in an individual might overcome, at least in part, the consequences of a function-modifying mutation in one or another critical component. If

391 this turns out to be the case, it could open the door to a new adjuvant therapeutic approach.

392 While speculative for now, experimental models in which a hypothesis could be tested are 393 available (54-56).

In summary, an intact permeability barrier is required for healthy gastrointestinal

395 function. While cell-cell adhesion structures are well-known participants in effective permeability

396 control, the present study provides evidence that cell-matrix interactions are also important.

397 These studies show, furthermore, that a multi-mineral natural product has the capacity to 
stimulate the production of cell-matrix adhesion moieties and, concomitantly, to improve barrier control.

\section{DATA AVAILABILITY}

401

402

403

404

405

406

407

408

409

410

411

412

413

414

415

416

417

All relevant data are within the manuscript and its Supporting Information files. The mass spectrometry proteomics data are available on ProteomeXchange Consortium (PRIDE partner repository) - identifier PXD020244.

\section{COMPETING INTERESTS}

There is no conflict of interest to declare from any author. Marigot LTD (Cork, Ireland) has provided Aquamin ${ }^{\circledR}$ as a gift for use in the study. This does not, in any way, alter our adherence to PLOS ONE policies on sharing data and materials.

\section{FUNDING}

This study was supported by the National Institutes of Health (NIH) grant CA201782 including supplemental funding through the Office of Dietary Supplements to JV and by an MCubed (University of Michigan) grant to MNA.

\section{ACKNOWLEDGMENTS}

We thank Marigot LTD (Cork, Ireland) for providing Aquamin ${ }^{\circledR}$ as a gift. We thank the Microscopy and Imaging Laboratory (MIL) for help with confocal fluorescence microscopy. We thank the Translational Tissue Modeling Laboratory (TTML) at the University of Michigan for help with colon organoid propagation and help with the TEER assay. We also thank the Proteomics Resource Facility (Pathology Department) for help with proteomic data acquisition. 


\section{REFERENCES}

1. Salim SY, Söderholm JD. Importance of disrupted intestinal barrier in inflammatory bowel diseases. Inflamm Bowel Dis. 2011;17(1):362-81. doi: 10.1002/ibd.21403. PMID: 20725949.

2. Antoni L, Nuding S, Wehkamp J, Stange EF. Intestinal barrier in inflammatory bowel disease. World J Gastroenterol. 2014;20(5):1165-79. doi: 10.3748/wjg.v20.i5.1165. PMID: 24574793.

3. Lee JY, Wasinger VC, Yau YY, Chuang E, Yajnik V, Leong RW. Molecular Pathophysiology of Epithelial Barrier Dysfunction in Inflammatory Bowel Diseases. Proteomes. 2018;6(2):17. doi: 10.3390/proteomes6020017. PMID: 29614738.

4. Vivinus-Nébot M, Frin-Mathy G, Bzioueche H, Dainese R, Bernard G, Anty R, et al. Functional bowel symptoms in quiescent inflammatory bowel diseases: role of epithelial barrier disruption and low-grade inflammation. Gut. 2014;63(5):744-52. doi: 10.1136/gutjnl-2012-304066. PMID: 23878165.

5. Pearson AD, Eastham EJ, Laker MF, Craft AW, Nelson R. Intestinal permeability in children with Crohn's disease and coeliac disease. Br Med J (Clin Res Ed). 1982;285(6334):20-1. doi: 10.1136/bmj.285.6334.20. PMID: 6805795.

6. Dunlop SP, Hebden J, Campbell E, Naesdal J, Olbe L, Perkins AC, et al. Abnormal intestinal permeability in subgroups of diarrhea-predominant irritable bowel syndromes. Am J Gastroenterol. 2006;101(6):1288-94. doi: 10.1111/j.1572-0241.2006.00672.x. PMID: 16771951.

7. Luissint AC, Parkos CA, Nusrat A. Inflammation and the Intestinal Barrier: LeukocyteEpithelial Cell Interactions, Cell Junction Remodeling, and Mucosal Repair. Gastroenterology. 2016;151(4):616-32. doi: 10.1053/j.gastro.2016.07.008. PMID: 27436072.

8. Flügel A, Schulze-Koops H, Heesemann J, Kühn K, Sorokin L, Burkhardt H, et al. Interaction of enteropathogenic Yersinia enterocolitica with complex basement membranes and the extracellular matrix proteins collagen type IV, laminin-1 and -2, and nidogen/entactin. J Biol Chem. 1994;269(47):29732-8. PMID: 7961965.

9. Moreira AP, Texeira TF, Ferreira AB, Peluzio Mdo C, Alfenas Rde C. Influence of a highfat diet on gut microbiota, intestinal permeability and metabolic endotoxaemia. $\mathrm{Br} \mathrm{J}$ Nutr. 2012;108(5):801-9. doi: 10.1017/S0007114512001213. PMID: 22717075.

10. Thaiss CA, Levy M, Grosheva I, Zheng D, Soffer E, Blacher E, et al. Hyperglycemia drives intestinal barrier dysfunction and risk for enteric infection. Science. 2018;359(6382):1376-1383. doi: 10.1126/science.aar3318. PMID: 29519916.

11. Meddings JB, Swain MG. Environmental stress-induced gastrointestinal permeability is mediated by endogenous glucocorticoids in the rat. Gastroenterology. 2000;119(4):1019-28. doi: 10.1053/gast.2000.18152. PMID: 11040188.

12. Clayburgh DR, Shen L, Turner JR. A porous defense: the leaky epithelial barrier in intestinal disease. Lab Invest. 2004;84(3):282-91. doi: 10.1038/labinvest.3700050. PMID: 14767487.

13. Turner JR. Molecular basis of epithelial barrier regulation: from basic mechanisms to clinical application. Am J Pathol. 2006;169(6):1901-9. doi: 10.2353/ajpath.2006.060681. PMID: 17148655.

14. Shen L, Su L, Turner JR. Mechanisms and functional implications of intestinal barrier defects. Dig Dis. 2009;27(4):443-9. doi: 10.1159/000233282. PMID: 19897958. 
15. Turner JR. Intestinal mucosal barrier function in health and disease. Nat Rev Immunol. 2009;9(11):799-809. doi: 10.1038/nri2653. PMID: 19855405.

16. Camilleri M, Madsen K, Spiller R, Greenwood-Van Meerveld B, Verne GN. Intestinal barrier function in health and gastrointestinal disease. Neurogastroenterol Motil. 2012;24(6):503-12. doi: 10.1111/j.1365-2982.2012.01921.x. PMID: 22583600.

17. Cereijido M, Valdés J, Shoshani L, Contreras RG. Role of tight junctions in establishing and maintaining cell polarity. Annu Rev Physiol. 1998;60:161-77. doi: 10.1146/annurev.physiol.60.1.161. PMID: 9558459.

18. Aijaz S, Balda MS, Matter K. Tight junctions: molecular architecture and function. Int Rev Cytol. 2006;248:261-98. doi: 10.1016/S0074-7696(06)48005-0. PMID: 16487793.

19. Green KJ, Simpson CL. Desmosomes: new perspectives on a classic. J Invest Dermatol. 2007;127(11):2499-515. doi: 10.1038/sj.jid.5701015. PMID: 17934502.

20. Kowalczyk AP, Green KJ. Structure, function, and regulation of desmosomes. Prog Mol Biol TransI Sci. 2013;116:95-118. doi: 10.1016/B978-0-12-394311-8.00005-4. PMID: 23481192.

21. Adey WH, McKibbin DL. Studies on the maerl species Phymatolithon calcareum (Pallas) nov. comb. and Lithothamnium corallioides Crouan in the Ria de Vigo. Botanical Marina 1970;13:100-106.

22. Attili D, McClintock SD, Rizvi AH, Pandya S, Rehman H, Nadeem DM, et al. Calciuminduced differentiation in normal human colonoid cultures: Cell-cell / cell-matrix adhesion, barrier formation and tissue integrity. PLoS One. 2019;14(4):e0215122. doi: 10.1371/journal.pone.0215122. PMID: 30995271.

23. McClintock SD, Attili D, Dame MK, Richter A, Silvestri SS, Berner MM, et al. Differentiation of human colon tissue in culture: Effects of calcium on trans-epithelial electrical resistance and tissue cohesive properties. PLoS One. 2020;15(3):e0222058. doi: 10.1371/journal.pone.0222058. PMID: 32134920.

24. Aslam MN, McClintock SD, Attili D, Pandya S, Rehman H, Nadeem DM, et al. Ulcerative Colitis-Derived Colonoid Culture: A Multi-Mineral-Approach to Improve Barrier Protein Expression. Front Cell Dev Biol. 2020;8:577221. doi: 10.3389/fcell.2020.577221. PMID: 33330453.

25. Schmehl K, Florian S, Jacobasch G, Salomon A, Körber J. Deficiency of epithelial basement membrane laminin in ulcerative colitis affected human colonic mucosa. Int $\mathrm{J}$ Colorectal Dis. 2000;15(1):39-48. doi: 10.1007/s003840050006. PMID: 10766090.

26. Verbeke S, Gotteland M, Fernández M, Bremer J, Ríos G, Brunser O. Basement membrane and connective tissue proteins in intestinal mucosa of patients with coeliac disease. J Clin Pathol. 2002;55(6):440-5. doi: 10.1136/jcp.55.6.440. PMID: 12037027.

27. Bouatrouss Y, Herring-Gillam FE, Gosselin J, Poisson J, Beaulieu JF. Altered expression of laminins in Crohn's disease small intestinal mucosa. Am J Pathol. 2000;156(1):45-50. doi: 10.1016/S0002-9440(10)64704-9. PMID: 10623652.

28. Spenlé C, Lefebvre O, Lacroute J, Méchine-Neuville A, Barreau F, Blottière HM, et al. The laminin response in inflammatory bowel disease: protection or malignancy? PLoS One. 2014;9(10):e111336. doi: 10.1371/journal.pone.0111336. PMID: 25347196.

29. Timpl R, Tisi D, Talts JF, Andac Z, Sasaki T, Hohenester E. Structure and function of laminin LG modules. Matrix Biol. 2000;19(4):309-17. doi: 10.1016/s0945053x(00)00072-x. PMID: 10963991.

30. Colognato $H$, Yurchenco PD. Form and function: the laminin family of heterotrimers. Dev Dyn. 2000;218(2):213-34. PMID: 10842354. 
31. Turck N, Gross I, Gendry P, Stutzmann J, Freund JN, Kedinger M, et al. Laminin isoforms: biological roles and effects on the intracellular distribution of nuclear proteins in intestinal epithelial cells. Exp Cell Res. 2005;303(2):494-503. doi: 10.1016/j.yexcr.2004.10.025. PMID: 15652360.

32. Gonzales M, Haan K, Baker SE, Fitchmun M, Todorov I, Weitzman S, et al. A cell signal pathway involving laminin-5, alpha3beta1 integrin, and mitogen-activated protein kinase can regulate epithelial cell proliferation. Mol Biol Cell. 1999;10(2):259-70. doi: 10.1091/mbc.10.2.259. PMID: 9950675.

33. Miyoshi $H$, Stappenbeck TS. In vitro expansion and genetic modification of gastrointestinal stem cells in spheroid culture. Nat Protoc. 2013;8(12):2471-82. doi: 10.1038/nprot.2013.153. PMID: 24232249.

34. Zou WY, Blutt SE, Crawford SE, Ettayebi K, Zeng XL, Saxena K, et al. Human Intestinal Enteroids: New Models to Study Gastrointestinal Virus Infections. Methods Mol Biol. 2019;1576:229-247. doi: 10.1007/7651_2017_1. PMID: 28361480.

35. Baker SE, Hopkinson SB, Fitchmun M, Andreason GL, Frasier F, Plopper G, et al. Laminin-5 and hemidesmosomes: role of the alpha 3 chain subunit in hemidesmosome stability and assembly. J Cell Sci. 1996;109 (Pt 10):2509-20. PMID: 8923212.

36. Green KJ, Jones JC. Desmosomes and hemidesmosomes: structure and function of molecular components. FASEB J. 1996;10(8):871-81. doi: 10.1096/fasebj.10.8.8666164. PMID: 8666164.

37. Suzuki N, Yokoyama F, Nomizu M. Functional sites in the laminin alpha chains. Connect Tissue Res. 2005;46(3):142-52. doi: 10.1080/03008200591008527. PMID: 16147852.

38. Chidgey, M. Plakin Proteins, Hemidesmosomes and Human Disease. In eLS, (Ed.). John Wiley \& Sons. 2012. https://doi.org/10.1002/9780470015902.a0024527

39. Has C. Hemidesmosomes: how much plakins do they need? Exp Dermatol. 2016;25(4):263-4. doi: 10.1111/exd.12939. PMID: 26740080.

40. Polari L, Alam CM, Nyström JH, Heikkilä T, Tayyab M, Baghestani S, et al. Keratin intermediate filaments in the colon: guardians of epithelial homeostasis. Int $\mathrm{J}$ Biochem Cell Biol. 2020;129:105878. doi: 10.1016/j.biocel.2020.105878. PMID: 33152513.

41. Zupancic T, Stojan J, Lane EB, Komel R, Bedina-Zavec A, Liovic M. Intestinal cell barrier function in vitro is severely compromised by keratin 8 and 18 mutations identified in patients with inflammatory bowel disease. PLoS One. 2014;9(6):e99398. doi: 10.1371/journal.pone.0099398. PMID: 24915158.

42. Corfe BM, Majumdar D, Assadsangabi A, Marsh AM, Cross SS, Connolly JB, et al. Inflammation decreases keratin level in ulcerative colitis; inadequate restoration associates with increased risk of colitis-associated cancer. BMJ Open Gastroenterol. 2015;2(1):e000024. doi: 10.1136/bmjgast-2014-000024. PMID: 26462276.

43. De Arcangelis $A$, Hamade $H$, Alpy $F$, Normand $S$, Bruyère $E$, Lefebvre $O$, et al. Hemidesmosome integrity protects the colon against colitis and colorectal cancer. Gut. 2017;66(10):1748-1760. doi: 10.1136/gutjnl-2015-310847. PMID: 27371534.

44. Stutzmann J, Bellissent-Waydelich A, Fontao L, Launay JF, Simon-Assmann P. Adhesion complexes implicated in intestinal epithelial cell-matrix interactions. Microsc Res Tech. 2000;51(2):179-90. PMID: 11054868.

45. Aslam MN, Bassis CM, Bergin IL, Knuver K, Zick SM, Sen A, et al. A Calcium-Rich Multimineral Intervention to Modulate Colonic Microbial Communities and Metabolomic Profiles in Humans: Results from a 90-Day Trial. Cancer Prev Res (Phila). 2020;13(1):101-116. doi: 10.1158/1940-6207.CAPR-19-0325. PMID: 31771942. 
46. Aslam MN, McClintock SD, Jawad-Makki MAH, Knuver K, Ahmad HM, Basrur V, et al. A Multi-Mineral Intervention to Modulate Colonic Mucosal Protein Profile: Results from a 90-Day Trial in Human Subjects. Nutrients. 2021;13(3):939. doi: 10.3390/nu13030939. PMID: 33799486.

47. Sequeira IR, Lentle RG, Kruger MC, Hurst RD. Standardising the lactulose mannitol test of gut permeability to minimise error and promote comparability. PLoS One. 2014;9(6):e99256. doi: 10.1371/journal.pone.0099256. PMID: 24901524.

48. Balk EM, Adam GP, Langberg VN, Earley A, Clark P, Ebeling PR, et al; International Osteoporosis Foundation Calcium Steering Committee. Global dietary calcium intake among adults: a systematic review. Osteoporos Int. 2017;28(12):3315-3324. doi: 10.1007/s00198-017-4230-x. PMID: 29026938.

49. U.S. Department of Health and Human Services; U.S. Department of Agriculture. 20152020 Dietary Guidelines for Americans. December 2015, 8th ed.; U.S. Department of Health and Human Services: Washington, DC, USA; U.S. Department of Agriculture: Washington, DC, USA, 2015. Available online: https://health.gov/our-work/food-andnutrition/2015-2020-dietary-guidelines/. (accessed on June 5, 2021).

50. Fine JD, Eady RA, Bauer EA, Bauer JW, Bruckner-Tuderman L, Heagerty A, et al. The classification of inherited epidermolysis bullosa (EB): Report of the Third International Consensus Meeting on Diagnosis and Classification of EB. J Am Acad Dermatol. 2008;58(6):931-50. doi: 10.1016/j.jaad.2008.02.004. PMID: 18374450.

51. Sachsenberg-Studer EM, Runne U, Wehrmann T, Wolter M, Kriener S, Engels K, et al. Bullous colon lesions in a patient with bullous pemphigoid. Gastrointest Endosc. 2001;54(1):104-8. doi: 10.1067/mge.2001.115472. PMID: 11427857.

52. Seo JW, Park J, Lee J, Kim MY, Choi HJ, Jeong HJ, Lee JW, Jung SY, Kim WK. A case of pemphigus vulgaris associated with ulcerative colitis. Intest Res. 2018;16(1):147-150. doi: 10.5217/ir.2018.16.1.147. PMID: 29422810.

53. Chen YJ, Juan CK, Chang YT, Wu CY, Ho HJ, Tseng HC. Association between inflammatory bowel disease and bullous pemphigoid: a population-based case-control study. Sci Rep. 2020;10(1):12727. doi: 10.1038/s41598-020-69475-0. PMID: 32728039.

54. Natsuga K, Shinkuma S, Nishie W, Shimizu H. Animal models of epidermolysis bullosa. Dermatol Clin. 2010;28(1):137-42. doi: 10.1016/j.det.2009.10.016. PMID: 19945627.

55. Bruckner-Tuderman L, McGrath JA, Robinson EC, Uitto J. Animal models of epidermolysis bullosa: update 2010. J Invest Dermatol. 2010;130(6):1485-8. doi: 10.1038/jid.2010.75. PMID: 20463671.

56. Heimbach L, Li N, Diaz A, Liu Z. Experimental animal models of bullous pemphigoid. G Ital Dermatol Venereol. 2009 Aug;144(4):423-31. PMID: 19755945. 


\section{FIGURE LEGENDS}

Fig 1. Transepithelial electrical resistance in differentiation medium. A: Time-dependent changes in TEER values. Values shown are means and standard deviations based on four separate experiments with 4 samples (individual membranes) per data point at each time-point in each experiment. Insert: Confocal fluorescent microscopic (max-projected) images of membranes stained after the day- 2 and day- 5 readings with antibody to occludin and with DAPI.

607 Scale bar $=50 \mu \mathrm{m}$. B: Effects of anti-laminin antibody on TEER values. Values shown are 608 means and standard deviations based on two separate experiments with 4 samples (individual 609 membranes) per data point at each time-point in each experiment. Insert: hematoxylin and 610 eosin-stained images of TEER membranes from IgG-treated and anti-laminin-treated wells.

611 Arrows in the anti-laminin-treated image show areas where cell detachment from the underlying

612 TEER membrane was visible. Scale bar $=100 \mu \mathrm{m}$ (small) and $50 \mu \mathrm{m}$ (Large).

Fig 2. Effects of Aquamin ${ }^{\circledR}$ on expression of proteins involved in cell-matrix interactions. generating the data sets were described in detail in past reports $(22,24)$. Data for individual

617 proteins were compared for statistical differences using the Student t-test. Asterisk $\left(^{*}\right)$ indicates

618 a difference from control at $p<0.05$. B: Western blotting. Protein isolated from each condition

619 was assessed for laminin $\beta 1$ expression by western blotting. $10 \mu \mathrm{g}$ of protein from each

620 condition was used. $\beta$-actin was assessed in parallel. Band quantitation was done using

621 ImageJ software.

622 Fig 3. Transepithelial electrical resistance in growth medium-KGM Gold with or without Aquamin $^{\circledR}$ and with or without anti-laminin antibody. TEER values shown are means and standard deviations based on three separate experiments with 4 samples (individual 
on TEER were assessed. Data were compared for statistical differences using ANOVA followed

627 by unpaired-group comparisons. Asterisk $\left(^{*}\right)$ above the open Aquamin ${ }^{\circledR}$ bar indicates a

628 difference from control at $p<0.05$. Asterisks $\left({ }^{*}\right)$ above the closed bars indicates difference from

629 respective IgG control at $p<0.05$.

Fig 4. Colon organoid cohesion in growth medium-KGM Gold: Effect of anti-laminin

631 antibody. Colon organoids were maintained for 14 days in growth medium-KGM Gold with and

632 without anti-laminin. At the end of the incubation period, cell-cell cohesion was assessed.

633 Values shown represent change in mean ( $( \pm S D)$ surface area of individual colonoids based on

634 two separate experiments with a minimum of 53 to 104 colonoids assessed individually per

635 treatment group in both pre- and post-harvest cultures. Data were compared for statistical

636 differences using ANOVA followed by unpaired-group comparisons. While the decrease in

637 organoid size between post-harvest and pre-harvest groups were statistically significant with

638 either IgG or anti-laminin, the differences between anti-laminin and IgG were not different.

639 Inset: Representative examples of pre-harvest and post-harvest cultures. Scale bar $=200 \mu \mathrm{m}$

640 Fig 5. Aquamin ${ }^{\circledR}$-responsive cell-matrix adhesion structures in the colon. Cartoon

641 depicting structures important to cell-matrix adhesion in the colon and components of those

642 structures that are responsive to Aquamin $^{\circledR}$ (shown in red). Based on the profile of proteins that

643 are induced by Aquamin ${ }^{\circledR}$, cell-matrix adhesion through both focal adhesions and

644 hemidesmosomes could be affected. 
Table 1. Top pathways associated with the proteins presented in Figure 2

Pathway name Submitted entities

\begin{tabular}{|l|l|}
\hline Type I hemidesmosome assembly & BP180;CD151;DST;LAMB3;LAMA3;LAMC2;PLEC \\
\hline Laminin interactions & LAMB3;LAMA1;LAMB2;LAMA3;LAMB1;LAMC2;LAMC1;NID1;HSPG2 \\
\hline $\begin{array}{l}\text { Extracellular matrix organization } \\
\text { interactions }\end{array}$ & $\begin{array}{l}\text { BP180;CD151;LAMB3;DST;LAMA1;LAMB2;LAMA3;LAMB1;LAMC2; } \\
\text { LAMC1;NID1;HSPG2;PLEC }\end{array}$ \\
\hline Degradation of the extracellular matrix & LAMB3;LAMA1;LAMB2;LAMA3;LAMB1;LAMC2;LAMC1;HSPG2 \\
\hline Collagen formation & BP180;CD151;DST;LAMB3;LAMA3;LAMC2;PLEC \\
\hline MET activates PTK2 signaling & LAMB3;LAMA1;LAMB2;LAMA3;LAMB1;LAMC2;LAMC1 \\
\hline $\begin{array}{l}\text { Assembly of collagen fibrils and other } \\
\text { multimeric structures }\end{array}$ & BP180;CD151;DST;LAMB3;LAMA3;LAMC2;PLEC \\
\hline MET promotes cell motility & LAMB3;LAMA1;LAMB2;LAMA3;LAMB1;LAMC2;LAMC1 \\
\hline ECM proteoglycans & LAMA1;LAMB2;LAMA3;LAMB1;LAMC1;HSPG2 \\
\hline Signaling by MET & LAMB3;LAMA1;LAMB2;LAMA3;LAMB1;LAMC2;LAMC1 \\
\hline Cell junction organization & BP180;CD151;DST;LAMB3;LAMA3;LAMC2;PLEC \\
\hline Cell-Cell communication & BP180;CD151;DST;LAMB3;LAMA3;LAMC2;PLEC \\
\hline Anchoring fibril formation & LAMB3;LAMA3;LAMC2 \\
\hline Signaling by Receptor Tyrosine Kinases & LAMB3;LAMA1;LAMB2;LAMA3;LAMB1;LAMC2;LAMC1 \\
\hline
\end{tabular}

The pathway analysis report was generated by Reactome database (v75) for species "Homo sapiens. 
Day 2

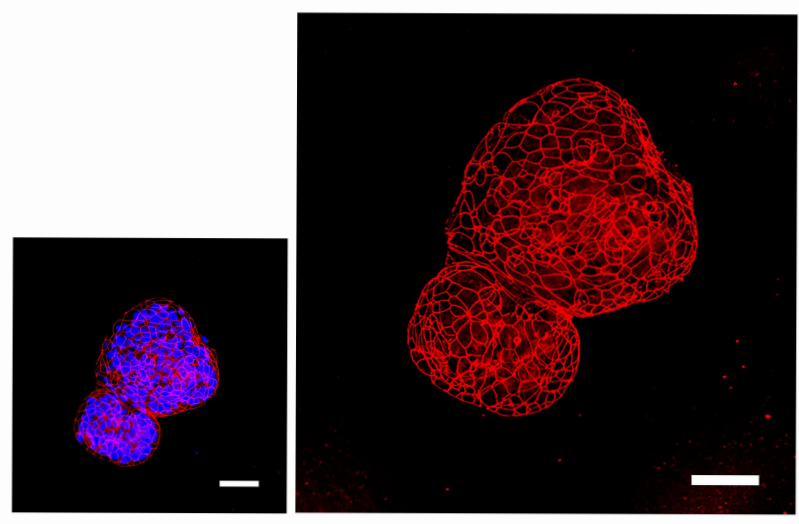

A.

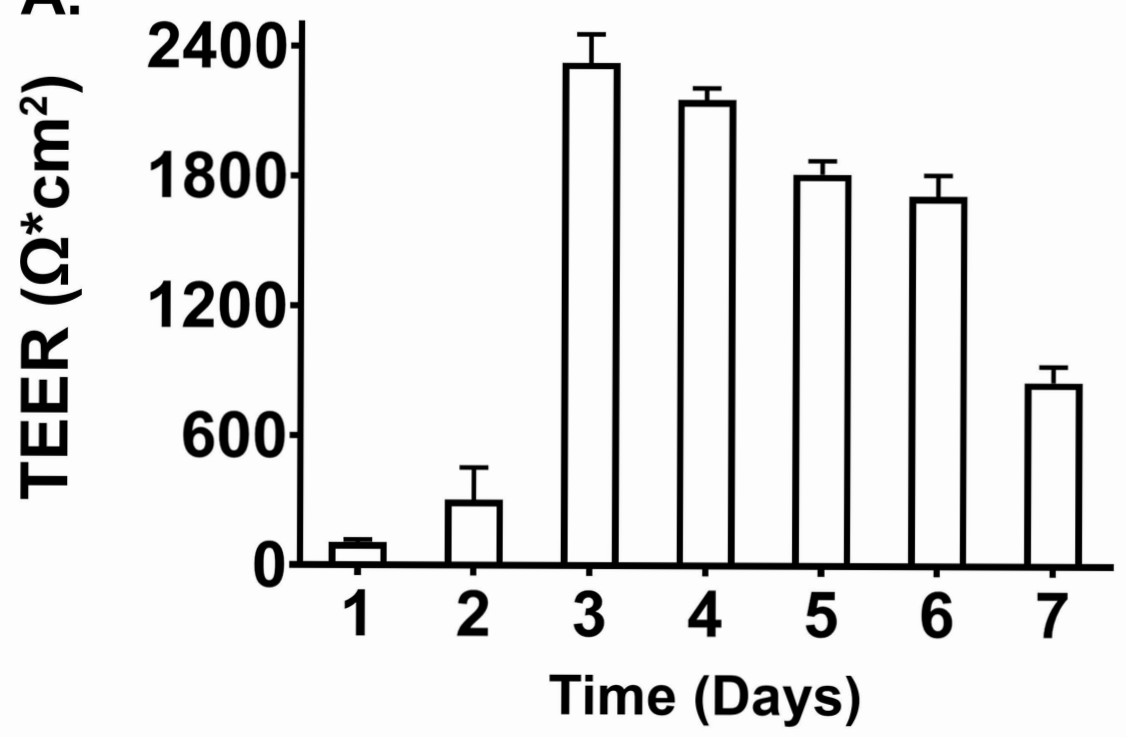

$\lg G$

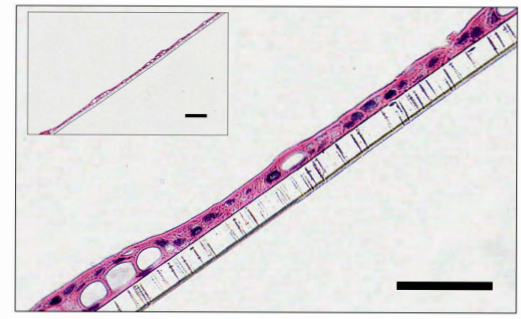

Anti-Laminin $\alpha 3$

B.

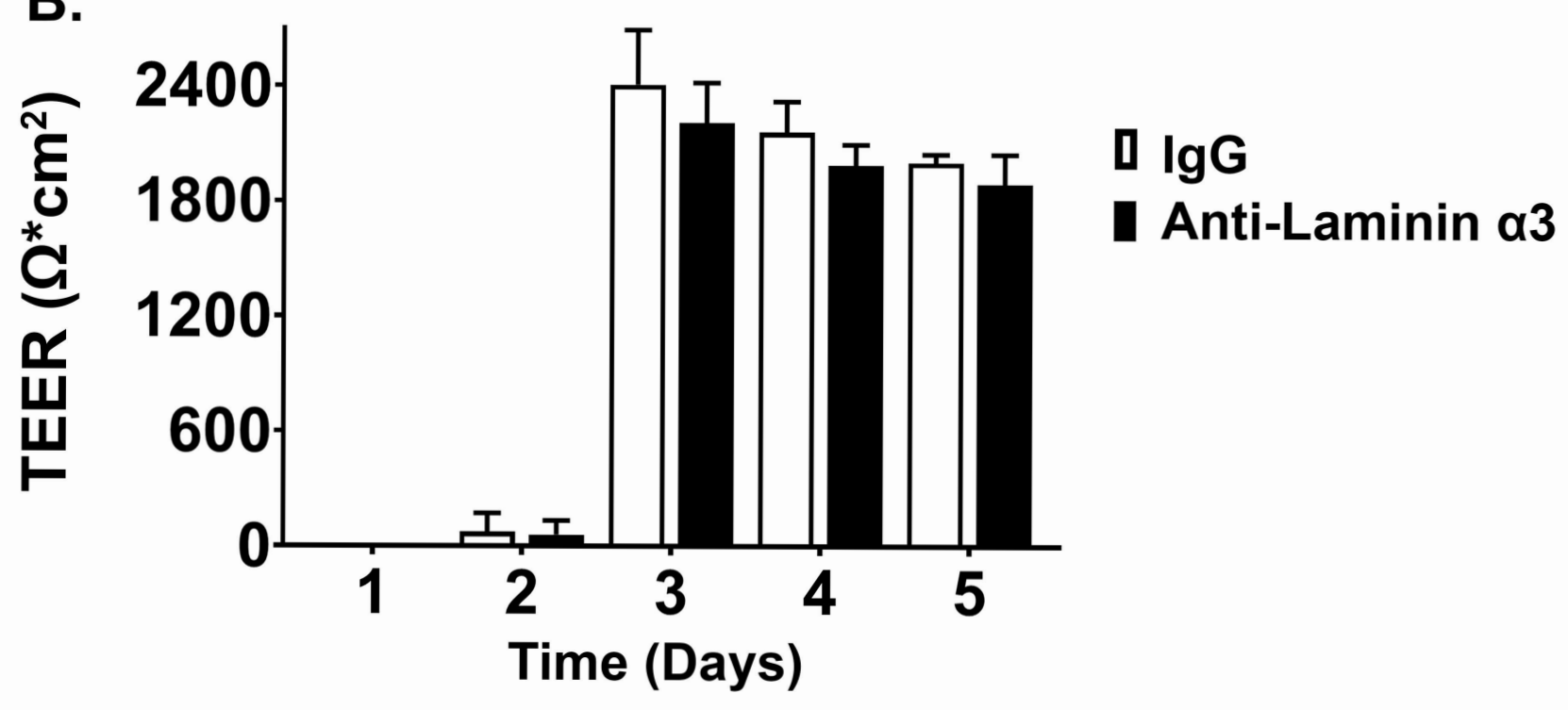


A.

Fig 2

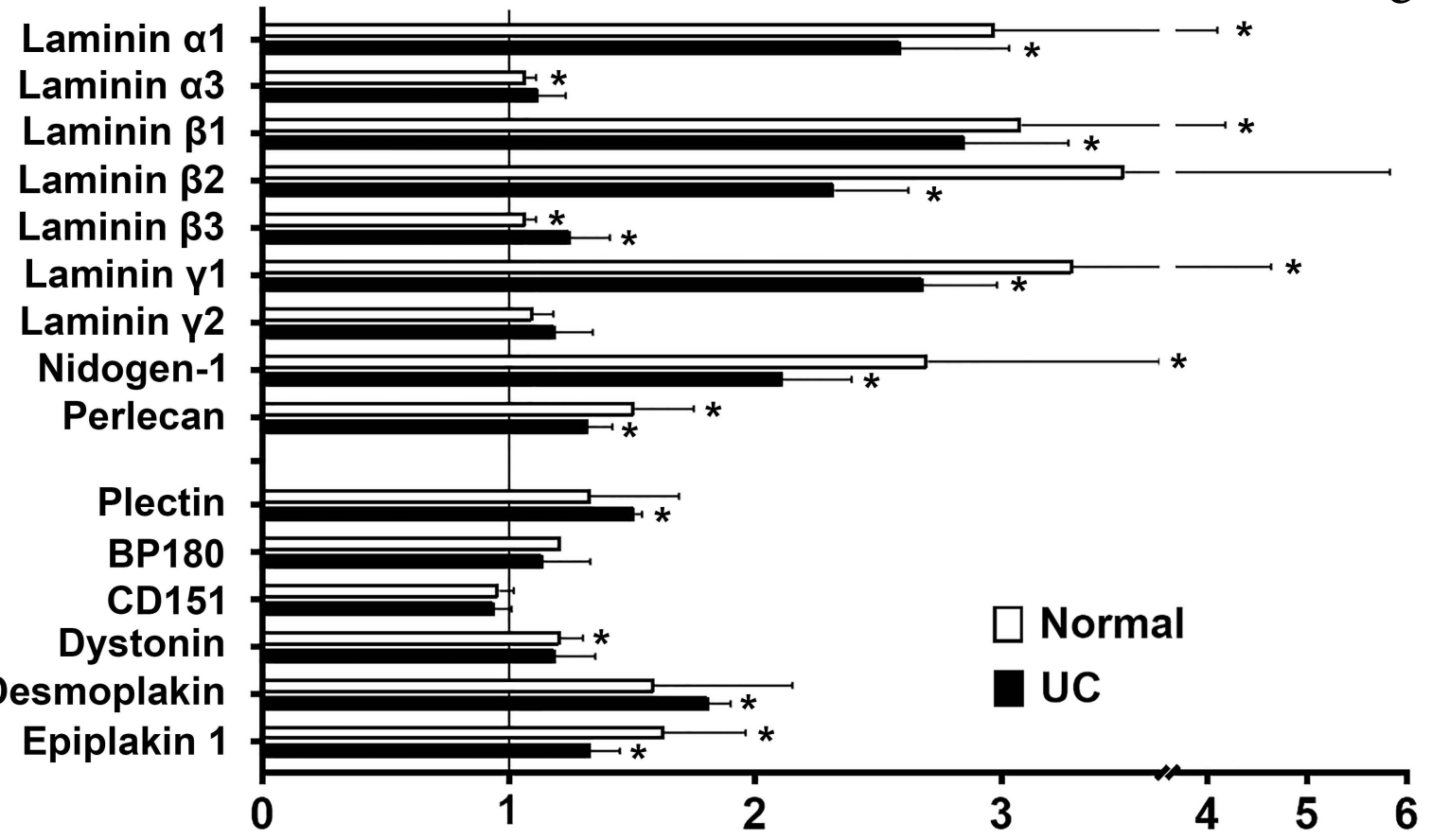

Relative Abundance (fold-change from control)

B.

Laminin $\beta 1 \quad \beta$-actin

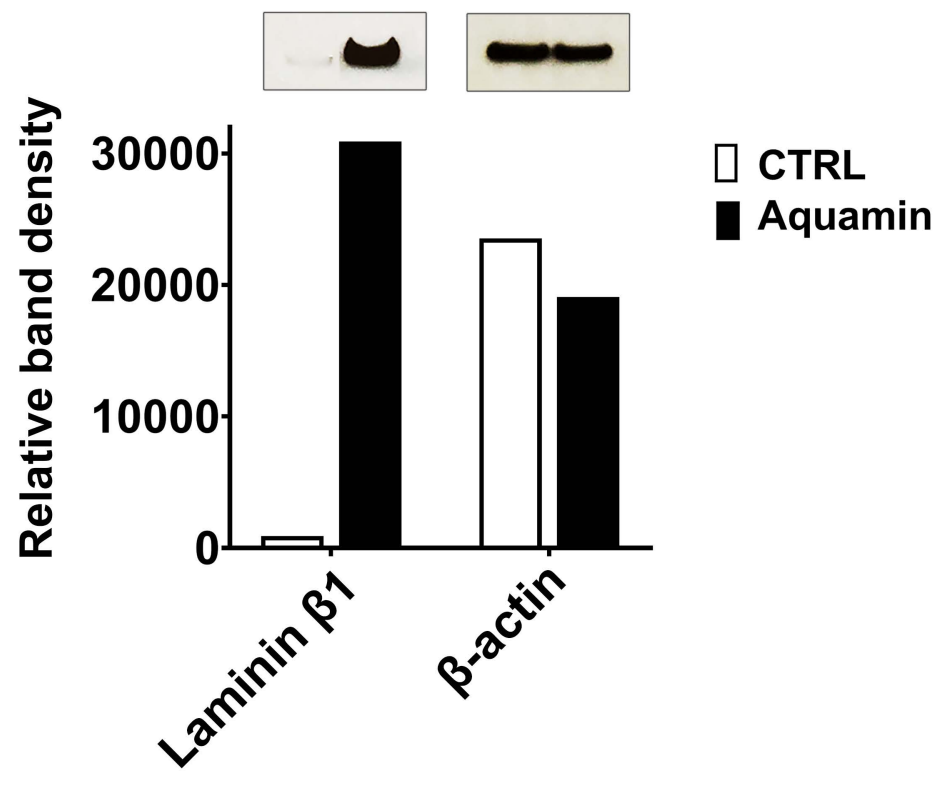


Fig 3

] lgG Anti-Laminin $\alpha 3$

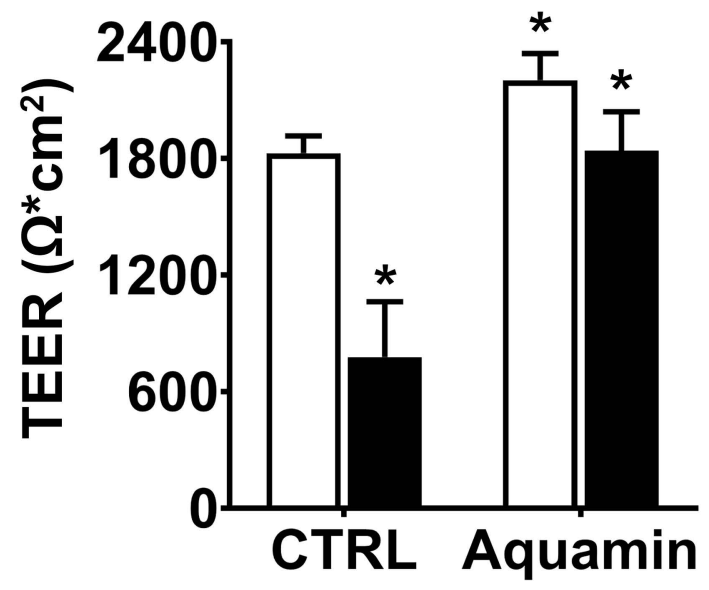


Fig 4

IgG Anti-Laminin $\alpha 3$
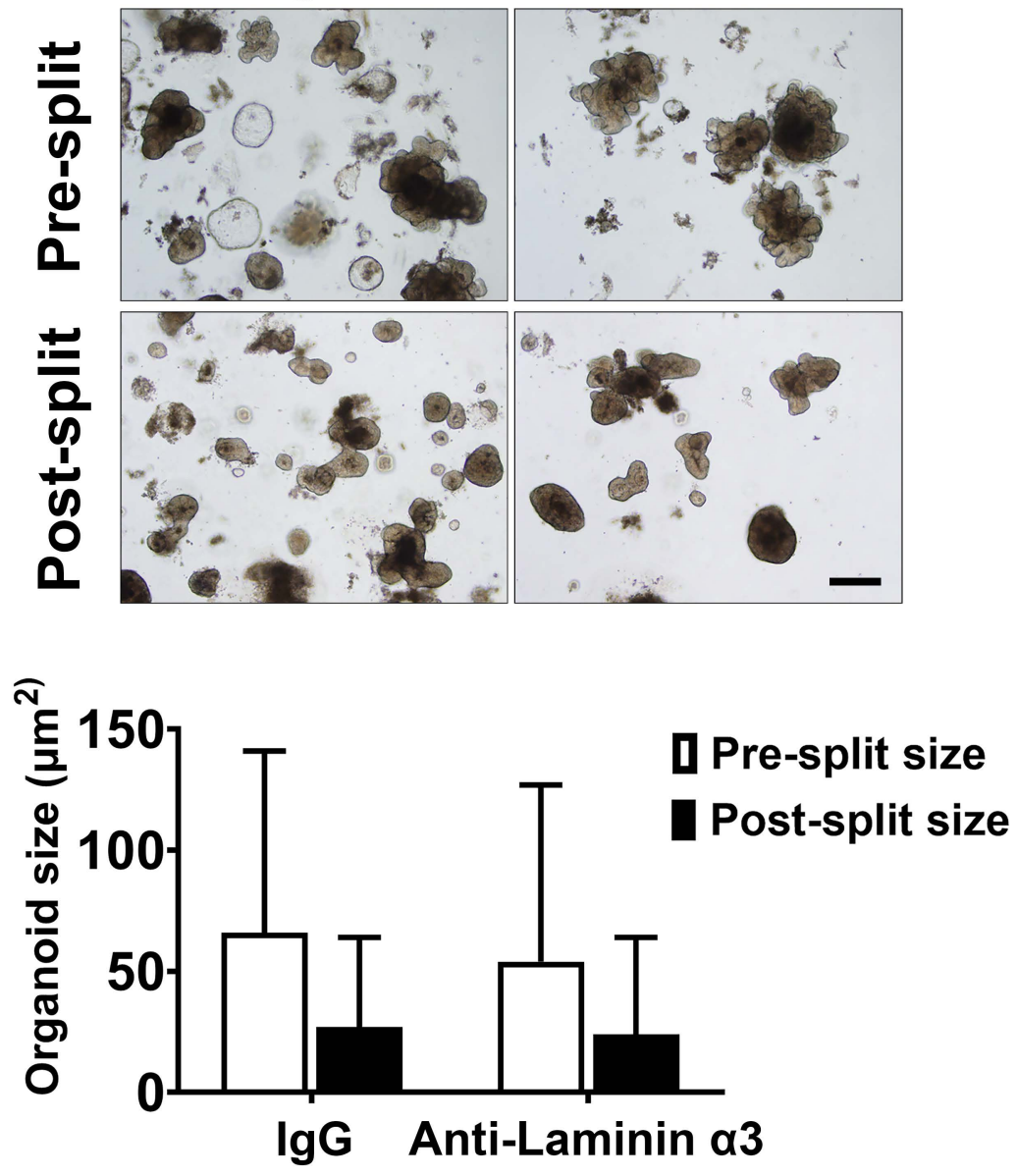


\section{Epithelial cell}

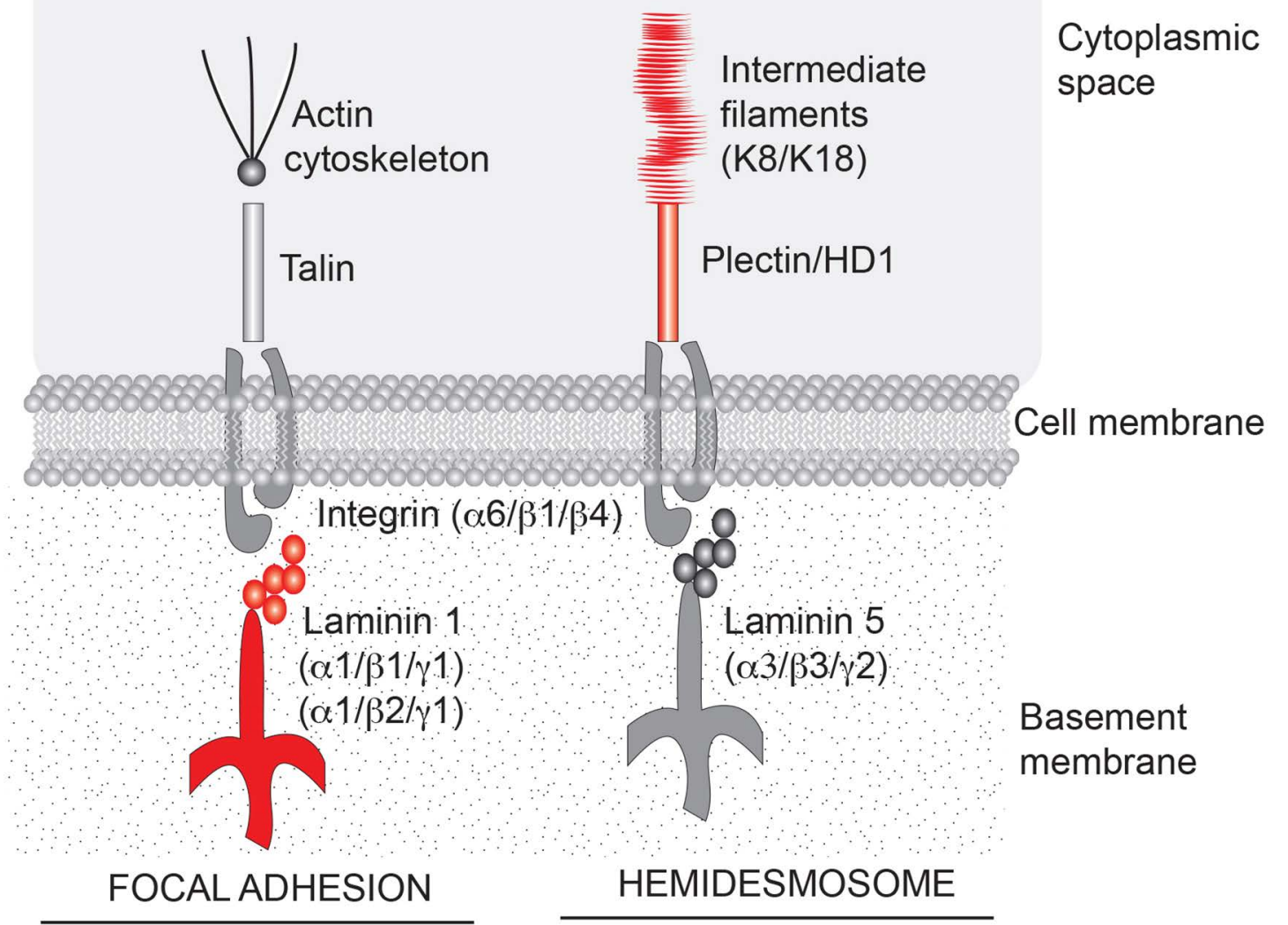

